ORIGINAL ARTICLE

\title{
How is deep vein thrombosis diagnosed and managed in UK and Australian emergency departments?
}

\author{
F C Sampson, S Goodacre, A-M Kelly, D Kerr
}

Emerg Med J 2005;22:780-782. doi: 10.1136/emj.2004.020610

Background: Recent research has identified technologies that may be of value in the diagnosis and management of deep vein thrombosis (DVT). We aimed to survey current practice in the United Kingdom (UK) and Australia to determine the extent to which these technologies have been implemented in these two healthcare systems.

Methods: We undertook a postal survey of 255 hospitals in the UK and 89 hospitals in Australia, requesting details of individual diagnostic tests, use of diagnostic algorithms, and management of DVT.

See end of article for authors' affiliations

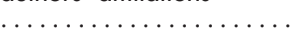

Correspondence to: F C Sampson, Medical Care Research Unit, University of Sheffield, Regent Court, 30 Regent Street, Sheffield, S1 4DA UK; f.c.sampson@ sheffield.ac.uk

Accepted for publication 6 December 2004 Results: We received replies from 186/255 UK hospitals (73\%) and 84/89 of Australian hospitals (94\%). Ultrasonography and laboratory based D-dimer were the most commonly available tests. We received 43 different algorithms from 51 hospitals. With only a very few exceptions, DVT diagnosis was ruled in by positive venography or positive ultrasound without venographic confirmation. By contrast a variety of different criteria were used to rule out DVT. Most algorithms used a combination of low clinical risk and negative D-dimer to rule out DVT, but some required all patients to receive ultrasound or venography. Few ruled out on the basis of low clinical risk or negative D-dimer alone. Low molecular weight heparins were overwhelmingly the treatment of choice for established DVT. Most departments $(214 / 264 ; 81 \%)$ offered outpatient treatment.

Conclusion: Recently developed technologies for the diagnosis and treatment of DVT have been widely implemented in the UK and Australia. Variation in practice, and thus presumably uncertainty, seems to be greatest in relation with the criteria used to rule out DVT.
$\mathrm{R}$ ecent developments in the diagnosis and management of deep vein thrombosis (DVT), such as clinical scoring, D-dimer, ${ }^{2}$ plethysmography, and ultrasound, ${ }^{3}$ and the development of outpatient treatment with low molecular weight heparin, ${ }^{4}$ mean that diagnosis can be completed and treatment initiated by the emergency department (ED). However, the variety of different diagnostic techniques available, and the sometimes conflicting evidence supporting them, may have led to uncertainty about the most appropriate diagnostic approach.

The uptake of technologies can vary between and within health services. ${ }^{5}$ Factors that may influence uptake include the quality of supporting evidence, dissemination of evidence to key decision makers, economic considerations, acceptability to patients, and the existence of professional and organisational barriers to change. We aimed to determine the extent of uptake of technologies in the diagnosis and management of suspected DVT in the United Kingdom (UK) and Australian healthcare systems. This will provide data to guide the development of health services and identify areas of uncertainty for future research.

\section{METHODS}

We undertook a postal survey of 255 EDs in the UK and 89 EDs in Australia in April 2003. These represent all major EDs in the UK (as defined by the British Association for Emergency Medicine Handbook) and all EDs accredited for emergency medicine training in Australia. The questionnaire was sent to the lead clinician or first named consultant and asked whether the department undertook diagnostic testing for DVT, whether they had a protocol or algorithm to guide diagnosis, which tests were routinely used in the diagnosis and management of DVT, and which were available by special request. We also asked about the standard initial treatment for patients with proven DVT and whether patients with proven DVT can be referred for outpatient treatment. If the ED did not undertake diagnostic testing we asked them to pass the survey onto the appropriate department. Up to two reminders were sent at three week intervals to nonrespondents. Data were analysed using SPSS for Windows, and are described by simple statistics and $\chi^{2}$ analysis. A $\mathrm{p}$ value of $<0.05$ was considered significant. Departments were asked to supply copies of any algorithms or protocols they used to guide diagnosis. We recorded all pathways of algorithms, including those that ruled out a diagnosis as well as those that were considered to provide definitive diagnosis of DVT.

\section{RESULTS}

One fifth of the UK EDs passed the survey on to other departments, usually general medicine or the medical assessment unit, where they were completed and returned, and have been included in the results. Fig 1 outlines the response rate and number of algorithms returned in each country. Fig 2 shows the individual diagnostic tests used. Ultrasound and laboratory based D-dimer were the most commonly available tests. There was little variation between the two countries in the tests used routinely, with the exception of computed tomography (CT) scanning, which was significantly more likely to be available in Australian EDs $(\mathrm{p}<0.005)$.

Only 51 algorithms were actually diagnostic algorithms, so analysis of algorithms was based upon data from 51/344 (15\%) of the surveyed hospitals. UK algorithms typically used combinations of D-dimer and ultrasound, whereas Australian algorithms typically used ultrasound with repeat scanning. Detailed analysis revealed that, of the 51 diagnostic

Abbreviations: $\mathrm{CT}$, computed tomography; DVT, deep vein thrombosis; $E D$, emergency department 


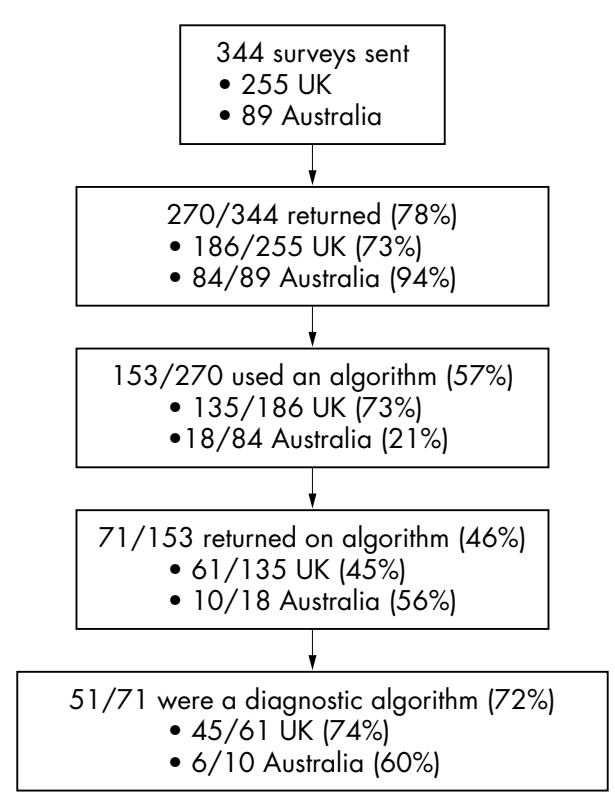

Figure 1 Flow chart of survey response and algorithm returns

algorithms returned, eight pairs of algorithms were used by two hospitals each and 35 were unique to their hospital. Thus there were 43 different algorithms available for detailed analysis.

Most algorithms (36/43) used clinical scoring systems. In 22 cases the published modified Wells criteria were used. ${ }^{1}$ In a further nine cases a score based on the Wells criteria, but with additional dimensions, was used. Additional dimensions included long haul air or coach travel (six protocols), previous DVT or pulmonary embolus (five protocols), use of hormone replacement therapy (five protocols), post partum or pregnancy (three protocols), and strong family history of DVT (three protocols). Five protocols mentioned use of a clinical score but did not specify details.

Table 1 shows the criteria used in the algorithms to diagnose DVT. With a few exceptions, the diagnosis of DVT was based upon a positive ultrasound result. Table 2 shows the criteria, other than a negative venogram or ultrasound result, used to rule out the diagnosis of DVT. These criteria showed less consistency, but most algorithms used combinations of D-dimer testing and clinical scoring. Few algorithms ruled out DVT on the basis of clinical score or D-dimer alone.

Variation was also apparent in the use of repeat scanning or venography to confirm a negative initial ultrasound scan. Eleven algorithms used a single negative ultrasound to rule out DVT, regardless of clinical risk or other test results, whereas 21 advocated either repeat scanning or venography,
Table 1 Criteria used to rule in deep vein thrombosis

\begin{tabular}{lc}
\hline Criterion & Number of algorithms \\
\hline Positive ultrasound scan & 38 \\
Positive venogram & 2 \\
Positive ultrasound, confirmed by venography & 1 \\
in low risk patients & 1 \\
Positive ultrasound or positive venometry* in \\
high risk patients \\
$\begin{array}{l}\text { Positive ultrasound or positive venometry in high } \\
\text { risk patients with a positive D-dimer }\end{array}$ \\
\hline *The venometer is a type of strain-gauge plethysmograph.
\end{tabular}

Table 2 Criteria, other than a negative venogram or ultrasound, used to rule out deep vein thrombosis

\begin{tabular}{lc}
\hline Criterion & Number of algorithms \\
\hline Negative D-dimer in low risk patients & 20 \\
Negative D-dimer in low or moderate risk & 4 \\
patients & 1 \\
Negative venometry in low risk patients & 4 \\
Negative D-dimer and venometry in low risk & 2 \\
patients & 2 \\
Negative D-dimer in low risk patients or & 1 \\
negative venometry in any patient & 2 \\
Negative D-dimer and venometry in any patient & 1 \\
Negative D-dimer in any patient & 8 \\
Low Wells score alone & \\
None, other than negative ultrasound or & \\
venogram &
\end{tabular}

dependent upon clinical risk or D-dimer results ( 6 clinical risk alone, $4 \mathrm{D}$-dimer alone, 11 both). This issue was not specified in 10 algorithms and three did not use D-dimer.

All six Australian algorithms used ultrasound and none used D-dimer. With one exception, clinical risk scoring was used to determine which patients required repeat scanning or venography following their ultrasound scan rather than to allow discharge without further testing.

\section{Management of proven DVT}

Subcutaneous low molecular weight heparin was the most commonly used standard initial treatment for patients with proven DVT, being used in $98 \%$ of both UK and Australian EDs (172/176 UK and 77/79 Australian). Intravenous, unfractionated heparin was used as standard initial treatment in the remaining $2 \%$ of hospitals in both countries. Patients with proven DVT were more likely to be referred for outpatient treatment in Australian EDs than UK EDs (94\%, $74 / 79 v 76 \%, 140 / 185 ; \mathrm{p}<0.001)$. In EDs where patients could receive outpatient treatment, over three quarters of patients

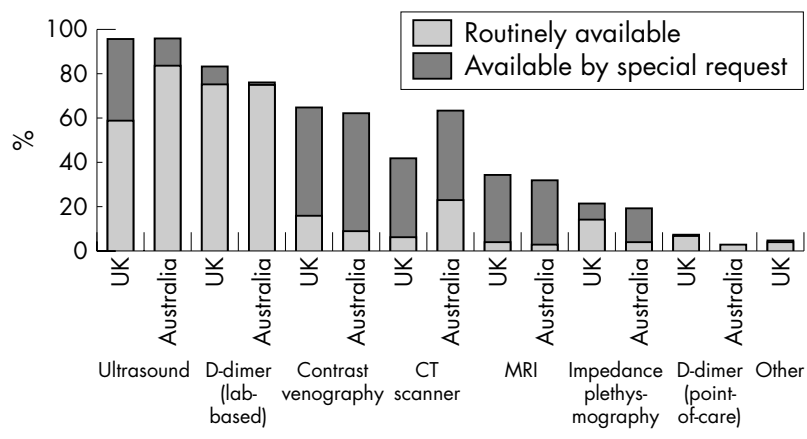

Figure 2 Tests used in the diagnosis and management of deep vein thrombosis (DVT) in UK and Australian emergency departments 
were estimated to be treated as outpatients (UK: 79\%, $\mathrm{n}=124$; Australia: $76 \%, \mathrm{n}=71$ ).

\section{DISCUSSION}

Non-invasive diagnostic assessment for DVT is widespread, particularly D-dimer and ultrasound, whereas there is a decreasing role for venography. Treatment with low molecular weight heparin is almost universal. Most departments provide outpatient treatment, and in these departments most patients are treated as outpatients. There appears to be some variation in how diagnostic modalities are used to rule out DVT. Although diagnosis of DVT was nearly always based upon a positive result of ultrasound (without venographic confirmation) or venography (as a sole test), numerous different criteria were used to rule out DVT, with many differences between algorithms. Evidence from cohort studies $^{6-9}$ indicates that treatment can safely be withheld from patients with a low clinical risk and negative D-dimer. This approach was used by most algorithms, but has by no means been adopted universally. This may reflect concerns about heterogeneity in the D-dimer assay, ${ }^{2}$ different thresholds for the definition of "safety", or may simply reflect variation in implementing recent research findings.

We surveyed hospitals in both the UK and Australia to increase the international relevance of our findings and allow comparison between healthcare systems. Although there was broad similarity in the tests available, we did identify some differences. CT scanning was more widely used in Australia whereas algorithms were more widely used in the UK. Allowing for the small number of Australian algorithms, there was some evidence that Australian strategies were more likely to ultrasound scan all patients and use clinical scoring to determine the need for repeat scanning whereas UK departments were more likely to use clinical scoring to identify patients who could be discharged on the basis of a negative D-dimer. This may reflect different approaches to access or funding for services between the two countries (particularly radiology services).

This study has a number of limitations that need to be appreciated. Only $15 \%$ of the departments surveyed returned a copy of an algorithm, so findings based upon analysis of algorithms need to be treated with caution and we must be careful about extrapolating conclusions to all hospitals. Being a postal survey, we can only report what hospitals say is being done, not what is actually being done. The true availability of tests may differ markedly from the reported availability. Likewise, estimates of the proportion treated reflect an impression rather than an actual value. Finally, to maximise the response rate we deliberately kept the survey short and simple. However, this cost some detail and we were therefore unable to report potentially useful data, such as the D-dimer assay used and whether ultrasound examination included distal veins.

Evidence of variation in practice may be used to identify areas of uncertainty for future research. We found little variation between the two countries, except for CT scanning. There was more variation in the range of different algorithms used and the criteria used to rule out DVT, although this finding is based on a relatively small number of algorithms. In the UK this may reflect a lack of national guidance in this area and some subjectivity in the judgment of what constitutes "safe" discharge without treatment. Whether patients should be discharged or undergo further testing should by determined by explicit and rational consideration of the risks, costs, and benefits of treating or not treating DVT. Future research should focus upon weighing these risks, costs, and benefits to develop guidelines for diagnosing DVT that reflect current evidence, national values, and priorities. ${ }^{10}$

\section{ACKNOWLEDGEMENTS}

We thank Suzanne Mason and Francis Morris for their advice in the development of the survey and the participating emergency departments for completing the questionnaire.

\section{Authors' affiliations}

F Sampson, S Goodacre, Medical Care Research Unit, University of Sheffield, Sheffield, United Kingdom

A-M Kelly, D Kerr, Joseph Epstein Centre for Emergency Medicine Research, Melbourne, Australia

Funding: This project was funded by the UK Health Technology Assessment R\&D Programme (project number 02/03/01). The views and opinions expressed therein are those of the authors and do not necessarily reflect those of the Department of Health.

Competing interests: none declared

\section{REFERENCES}

1 Wells PS, Anderson DR, Mormanis J, et al. Value of assessment of pre-test probability of deep vein thrombosis in clinical management. Lancet 1997;350:1795-8.

2 Kelly J, Rudd A, Lewis RR, et al. Plasma D-dimers in the diagnosis of venous thrombosis. Ann Intern Med 2002;162(7):747-56.

3 Kearon C, Julian JA, Newman TE, et al. Noninvasive diagnosis of deep venous thrombosis. Ann Intern Med 1998;128(8):663-77.

4 Gould MK, Dembitzer AD, Doyle RL, et al. Low-molecular-weight heparins compared with unfractionated heparin for treatment of acute deep venous thrombosis. Ann Intern Med 1999;130:800-9.

5 NHS Centre for Reviews and Dissemination. Effective health care: getting evidence into practice. Effective Health Care Bulletins 1999:5(1).

6 Kearon C, Ginsberg JS, Douketis J, et al. Management of suspected deep venous thrombosis in outpatients by using clinical assessment and D-dimer testing. Ann Intern Med 2001;135(2):108-1 1 .

7 Janes S, Ashford N. Use of a simplified clinical scoring system and D-dimer testing can reduce the requirement for radiology in the exclusion of deep vein thrombosis by over 20\%. Br J Haematol 200, 112(4):1079-82.

8 Bates SM, Kearon C, Crowther M, et al. A diagnostic strategy involving a quantitative latex D-dimer assay reliably excludes deep venous thrombosis. Ann Intern Med 2003;138(10):787-94.

9 Schutgens RE, Ackermark P, Haas FJ, et al. Combination of a normal $\mathrm{D}$-dimer concentration and a non-high pretest clinical probability score is a safe strategy to exclude deep venous thrombosis. Circulation 2001;107(4):593-7.

10 Raftery J. NICE: faster access to modern treatments? Analysis of guidance on health technologies. BMJ 2001;323(7324):1300-3 